\title{
High glucose reduces hepatic glycogenesis by suppression of microRNA-152
}

\author{
XIAOYUN ZHAO ${ }^{1}$, YONGGANG LU ${ }^{1}$, FENGHONG WANG ${ }^{1}$, LIN DOU ${ }^{2}$, LILIN WANG ${ }^{2}$, JUN GUO $^{2}$ and JIAN LI ${ }^{2}$ \\ ${ }^{1}$ Clinical Laboratory, Hebei General Hospital, Shijiazhuang, Hebei 050057; ${ }^{2}$ Key Laboratory of Geriatrics, \\ Beijing Institute of Geriatrics and Beijing Hospital, Ministry of Health, Beijing 100730, P.R. China
}

Received November 13, 2013; Accepted May 9, 2014

DOI: $10.3892 / \mathrm{mmr} .2014 .2426$

\begin{abstract}
MicroRNAs (miRNAs) are a class of non-coding 18-25-nucleotide endogenous RNA molecules. miRNAs act as specific gene silencers to regulate target gene expression at the posttranscriptional level, by base pairing to the 3'-untranslated region of the target mRNA. miR-152 is an miRNA that was originally identified in cancer cells, and was shown to be able to modulate the expression of specific oncogenes and tumor suppressor genes, leading to enhanced carcinoma growth. However, little is known regarding the role of miR-152 in the regulation of hepatic insulin resistance and glucose metabolism. In the present study, it was identified that the activation of AKT and glycogen synthase kinase 3 (GSK-3), and the expression levels of glycogen, were reduced in mouse NCTC 1469 hepatocytes and mouse primary hepatocytes, following exposure to $25 \mathrm{mM}$ glucose for $48 \mathrm{~h}$. Furthermore, it was demonstrated that high glucose levels suppressed the expression of miR-152 in hepatocytes. In order to further assess the effects of miR-152 on the glucose-induced reduction of glycogen synthesis and activation of AKT and GSK, miR-152 mimic and inhibitor were transfected into the NCTC 1469 cells, respectively. The transfection of the miR-152 inhibitor resulted in reduced expression of glycogen, accompanied by impaired phosphorylation of AKT and GSK in the NCTC 1469 cells treated with or without glucose. Conversely, upregulation of miR-152 by transfection of an miR-152 mimic reversed the glucose-induced decrease in glycogen synthesis and reduced AKT and GSK phosphorylation in hepatocytes. This indicated that miR-152 could modulate the AKT/GSK pathway and glycogen synthesis. In conclusion, to the best of our knowledge, this study was the first to indicate that high glucose impaired the activation of the AKT/GSK pathway and the synthesis of
\end{abstract}

Correspondence to: Professor Jian Li, Key Laboratory of Geriatrics, Beijing Institute of Geriatrics and Beijing Hospital, Ministry of Health, 1 Dahua Road, Dongdan, Beijing 100730, P.R. China

E-mail: lijian@bjhmoh.cn

Key words: glucose, microRNA-152, AKT/glycogen synthase kinase pathway, hepatic glycogenesis glycogen in mouse hepatocytes, in part through the downregulation of miR-152.

\section{Introduction}

Insulin resistance is a condition of impaired or diminished insulin sensitivity, in which the normal expression levels of insulin fail to induce the normal insulin response in the liver, adipose tissue and muscle cells. Insulin acts as a critical factor in the pathogenesis of type 2 diabetes and metabolic syndrome. Hepatic glucose metabolism is tightly controlled by circulating insulin, due to its inhibitory effects on gluconeogenesis and stimulatory effects on glycogenesis in the liver. In insulin resistant states, hepatic glycogen synthesis is impaired, which results in increased glucose production (1).

Insulin signaling is essential for the maintenance of hepatic glucose homeostasis. In stable states, glycogensynthase (GS) is phosphorylated by activated glycogen synthase kinase 3 (GSK-3), which causes the inhibition of glycogen synthesis. The phosphoinositide-3 kinase (PI3K)/AKT pathway is activated when hepatocytes respond to insulin, leading to the phosphorylation of GSK-3 and inactivation of GSK. This results in the generation of glycogen synthesis through activated GS (2-4). Hyperglycemia is regarded as a consequence of insufficient insulin secretion and insulin resistance. Several studies have identified that hyperglycemia can lead to an apparent improvement of insulin resistance in the liver (5). Glucotoxicity is a major cause of $\beta$-cell dysfunction that can lead to impaired insulin signaling action or insulin resistance in the liver, consistent with decreased glycogen levels in hepatocytes.

MicroRNAs (miRNAs) are a class of non-coding 18-25 nucleotide endogenous RNA molecules, which act as specific gene silencers to regulate the target gene expression at the posttranscriptional level by base pairing to the $3^{\prime}$ untranslated region of the target mRNA. Numerous studies have indicated that miRNAs have a critical regulatory role in various metabolic diseases, including diabetes mellitus, obesity and metabolic syndrome. miR-375, miR-29, miR-9 and Let-7 have been previously associated with regulating insulin secretion (6). miR-375 and miR-124a have additionally been shown to participate in pancreatic islet development (7) and $\beta$-cell differentiation (8). Although it has been reported that miR-181 may participate in the development of insulin resistance by the 
Table I. Sequences of primers used for reverse transcription.

Reverse-transcriptional primer (5'-3')

U6

miR-152
GTCGTATCCAGTGCAGGGTCCGAGGTATTCGCACTGGATACGACAAAATATG GTCGTATCCAGTGCAGGGTCCGAGGTATTCGCACTGGATACGACCCAAGT

miR, microRNA.

regulation of Sirtuin 1 expression at the translational level in hepatocytes, the mechanisms of miRNAs involved in hepatic insulin resistance remain unknown (9).

Decreased levels of miR-152 have been identified to accelerate the tumor growth of certain types of tumors (10). It has been reported that DNA methyltransferase-1 (DNMT-1) is a target of miR-152, and functions in the maintenance of DNA methylation $(8,11,12)$. In cancer cells, miR-152 was shown to directly modulate the expression of DNMT-1, which in turn could modulate the expression of specific oncogenes and tumor suppressor genes, leading to enhanced carcinoma growth. There is little known regarding the role of miR-152 in the regulation of hepatic insulin resistance and glucose metabolism. This study has provided for the first time, to the best of our knowledge, novel experimental evidence showing that high glucose levels impaired the activation of the AKT/GSK pathway and the synthesis of glycogen in hepatocytes, at least in part through the downregulation of miR-152.

\section{Materials and methods}

Cell culture. NCTC 1469 cells were derived from mouse liver cells (American Type Culture Collection, Manassas, VA, USA) and cultured in low-glucose Dulbecco's modified Eagle's medium (5 mmol/l glucose; Gibco-BRL, Carlsbad, CA, USA) supplemented with $10 \%$ horse serum (HyClone, Logan, UT, USA), $100 \mathrm{U} / \mathrm{ml}$ penicillin (Gibco-BRL) and $0.1 \mathrm{mg} / \mathrm{ml} \mathrm{strep-}$ tomycin (Gibco-BRL) at $37^{\circ} \mathrm{C}$ in a humidified atmosphere of $95 \% \mathrm{O}_{2}$ and $5 \% \mathrm{CO}_{2}$.

Isolation of mouse primary hepatocytes. Male C57BL/6J mice (age, 12 weeks) were provided by Peking University Health Science Center (originally purchased from Jackson Laboratory, Bar Harbor, ME, USA). All animal procedures were performed in accordance with the National Institutes of Health Animal Care and Use Guidelines. All animal protocols were approved by the Animal Ethics Committee at the Beijing Institute of Geriatrics (Beijing, China). Primary hepatocytes were isolated by a two-step collagenase perfusion $[0.2 \mathrm{mg} / \mathrm{ml}$ type IV collagenase (Sigma, St. Louis, MO, USA) in Hanks' balanced salt solution], as described previously (13). The hepatocytes were collected by centrifugation at $430 \mathrm{x} \mathrm{g}$ for $8 \mathrm{~min}$. Immediately after harvesting, the cells were suspended in pre-warmed William's E medium (Sigma) supplemented with $10 \%$ fetal bovine serum, $20 \mathrm{ng} / \mathrm{ml}$ dexamethasone (Sigma), ITS (containing $5 \mathrm{mg} / \mathrm{l}$ insulin, $5 \mathrm{mg} / \mathrm{l}$ trasferrin and $5 \mu \mathrm{g} / 1$ sodium selenate; Sigma) and $10 \mu \mathrm{g} / \mathrm{ml}$ gentamicin (Invitrogen Life Technologies, Carlsbad, CA, USA). Hepatocytes were plated in collagen-coated $25-\mathrm{cm}^{2}$ flasks at a density of $1 \times 10^{6}$ cells per flask.
Table II. Nucleotide of primers used for qPCR.

\begin{tabular}{ll}
\hline & Forward primer (5'-3') \\
\hline Universal primer & GTGCAGGGTCCGAGGT \\
U6 & GCGCGTCGTGAAGCGTTC \\
miR-152 & TCAGTGCATGACAGA \\
\hline
\end{tabular}

qPCR, quantitative polymerase chain reaction; miR, microRNA.

Transfection of miRNA mimic and inhibitor. The mimic and inhibitor of miR-152 were purchased from Genepharma (Shanghai, China). miRNA mimic and inhibitor controls were used as negative controls, respectively. Hiperfect Transfection Reagent (Qiagen, Hilden, Germany) was used for the transfection of the miR-152 mimic and inhibitor. The expression of miR-152 was detected by quantitative polymerase chain reaction (qPCR), $48 \mathrm{~h}$ after transfection.

RNA isolation and $q P C R$. Enriched miRNA was isolated using an miRNA isolation kit (Takara Bio Inc., Shiga, Japan). Stem-loop reverse transcription-PCR was performed on the samples to detect and quantify mature miRNA, using a stem-loop antisense primer mix (Tables I and II) and AMV transcriptase (Takara). The cDNA was routinely tested by qPCR based on the SYBR Green I method, according to the manufacturer's instructions (Takara). Amplification and detection of the specific products were performed according to the manufacturer's instructions using an ABI PRISM 7500 system (Applied Biosystems ${ }^{\circledR}$, Invitrogen Life Technologies). The U6 small nucleolar RNA was used as the house-keeping small RNA reference gene. The relative gene expression was normalized to the U6 small nucleolar RNA. Each reaction was performed in triplicate, and analysis was performed using the $2^{-\Delta \Delta C T}$ method.

Western blot analysis. Cell lysates (15-30 $\mu \mathrm{g}$ of protein) were separated by $10 \%$ SDS-PAGE, then transferred to a polyvinylidene fluoride membrane (Millipore, Billerica, MA, USA). The membranes were then blocked with $8 \%$ non-fat dry milk and probed with the primary antibodies at $4^{\circ} \mathrm{C}$ overnight. The blots were incubated with horseradish peroxidase-conjugated anti-IgG, followed by detection with enhanced chemiluminescence (Millipore). Antibodies against AKT, phosphorylated AKT $\left(\operatorname{Ser}^{473}\right)$, glycogen synthase kinase (GSK), and phosphorylated GSK $\left(\operatorname{Ser}^{9}\right)$ were purchased from Cell Signaling Technology, Inc., (Beverly, MA, USA). Antibodies to $\beta$-actin were obtained from Santa Cruz (Santa Cruz Biotechnology Inc., Santa Cruz, CA, USA). 
A

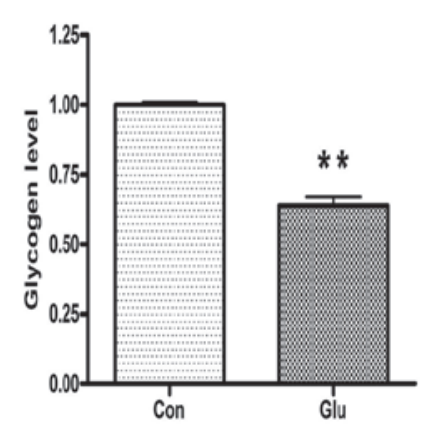

C

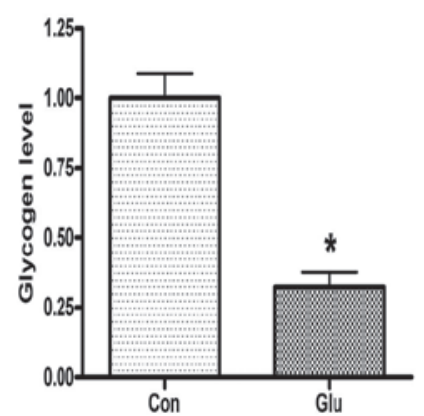

B

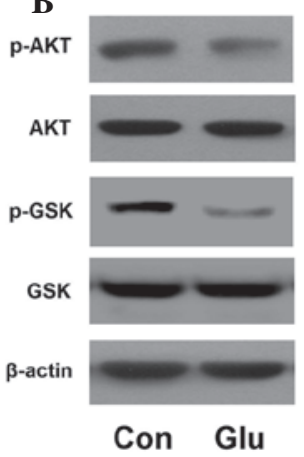

D

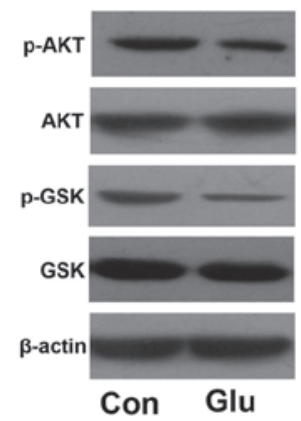

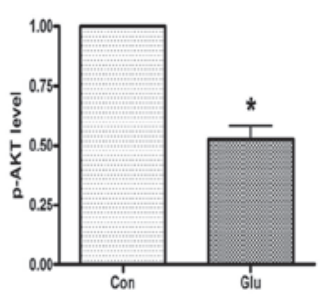
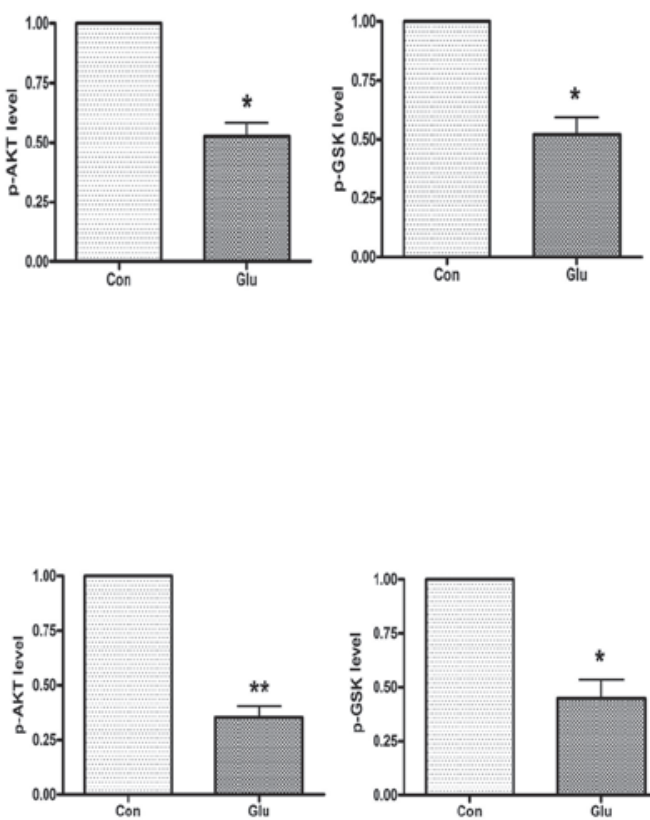

Figure 1. High glucose induced a reduction in glycogen synthesis in hepatocytes. Mouse NCTC 1469 hepatocytes were treated with 25 mM glucose for 48 h. (A) High glucose treatment significantly decreased the glycogen levels, and (B) reduced the phosphorylation of AKT and GSK in NCTC 1469 cells. (C and D) Mouse primary hepatocytes were treated with $25 \mathrm{mM}$ glucose for $48 \mathrm{~h}$. The glycogen levels and phosphorylation of AKT and GSK were also decreased in the mouse primary hepatocytes in response to high glucose treatment. Data represent the mean \pm standard error of the mean, $n=3$ independent experiments. ${ }^{*} \mathrm{P}<0.05$ and ${ }^{* *} \mathrm{P}<0.01$ vs. control. Con, control; Glu, $25 \mathrm{mM}$ glucose; p-, phosphorylated; GSK, glycogen synthase kinase.

Glycogen content measurement. Glycogen levels were measured in cells incubated with $1 \mathrm{nmol} / 1$ insulin (United States Biological, Salem, MA, USA), for $3 \mathrm{~h}$, using a Glycogen Assay kit (BioVision, Mountain View, CA, USA).

Statistical analysis. All values are represented as the mean \pm standard error of the mean, of the indicated number of measurements. One-way analysis of variance was used to determine statistical significance. $\mathrm{P}<0.05$ considered to indicate a statistically significant difference.

\section{Results}

High glucose levels induce a reduction of glycogen synthesis in hepatocytes through impairing phosphorylation of AKT and GSK. Mouse NCTC 1469 hepatocytes were treated with $25 \mathrm{mM}$ glucose for $48 \mathrm{~h}$ and the glycogen levels were measured. As shown in Fig. 1A, the high glucose treatment significantly decreased the expression levels of glycogen in NCTC 1469 cells. Furthermore, the phosphorylation of AKT and GSK was significantly reduced in the NCTC 1469 cells treated with $25 \mathrm{mM}$ glucose for $48 \mathrm{~h}$ (Fig. 1B). In order to further assess the effects of high glucose on glycogen synthesis, mouse primary hepatocytes were also treated with $25 \mathrm{mM}$ glucose for $48 \mathrm{~h}$. The results indicated that glycogen levels and phosphorylation of AKT and GSK were reduced in mouse primary hepatocytes in response to high glucose treatment (Fig. 1C and D), indicating that high glucose induced a reduction of glycogen synthesis
A

B
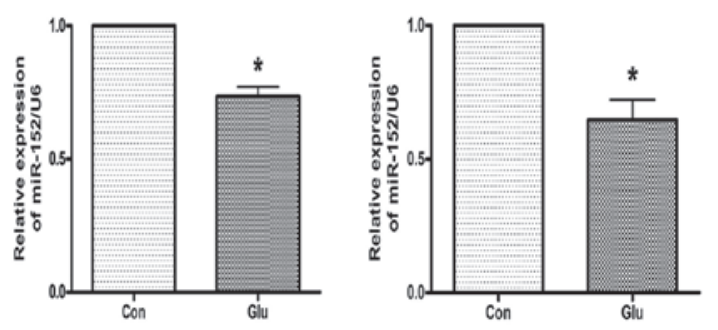

Figure 2. High glucose suppressed the expression of miR-152 in the hepatocytes. (A) As analyzed by quantitative polymerase chain reaction, the expression of miR-152 was downregulated in the NCTC 1469 cells treated with $25 \mathrm{mM}$ glucose for $48 \mathrm{~h}$. (B) High glucose suppressed the expression of miR-152 in the mouse primary hepatocytes. Data represent the mean \pm standard error of the mean, $\mathrm{n}=3$ independent experiments. ${ }^{*} \mathrm{P}<0.05$ vs. control. miR-152, microRNA-152; Con, control; Glu, 25 mM glucose.

in the hepatocytes, through impairing the phosphorylation of AKT and GSK.

High glucose suppresses the expression of miR-152 in hepatocytes. The effects of high glucose on the expression of miR-152 were investigated. As analyzed by qPCR, the expression of miR-152 was downregulated in NCTC 1469 cells treated with $25 \mathrm{mM}$ glucose for $48 \mathrm{~h}$ (Fig. 2A). High glucose additionally suppressed the expression of miR-152 in mouse primary hepatocytes (Fig. 2B). These data suggest that miR-152 may be involved in glucose-induced insulin resistance. 
A

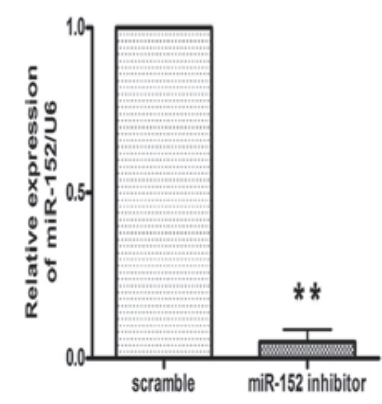

C

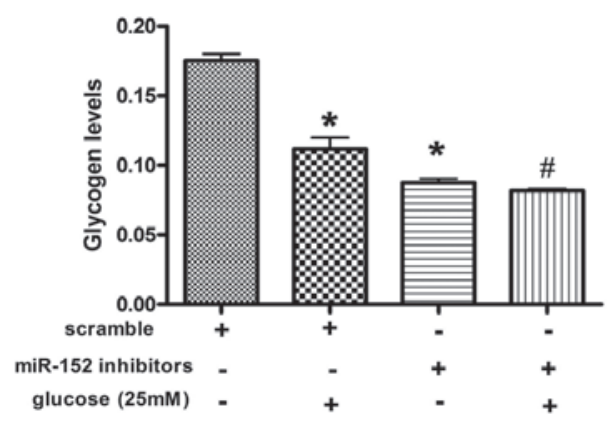

B

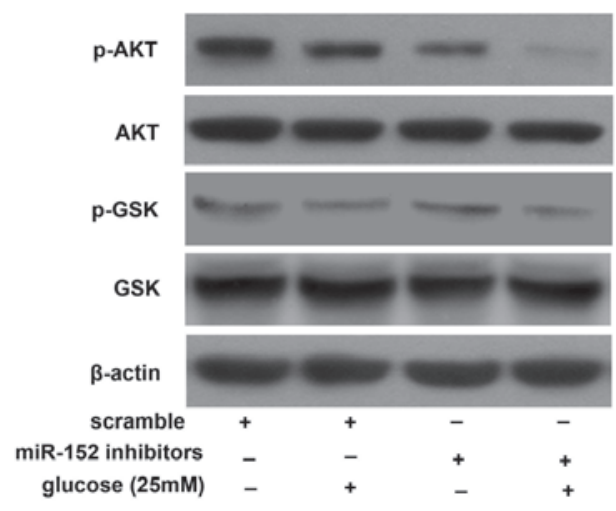

D

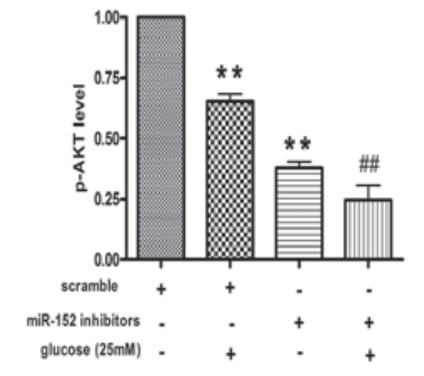

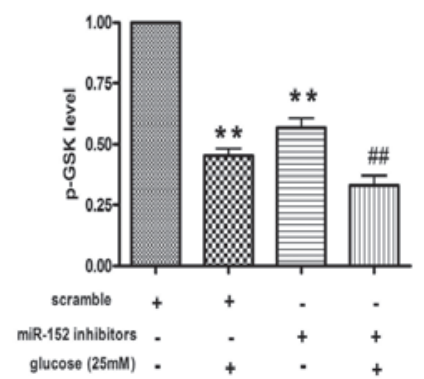

Figure 3. miR-152 inhibitor promotes a reduction in glycogen synthesis and impairment of AKT and GSK phosphorylation in the hepatocytes. (A) miR-152 inhibitor was transfected into the NCTC 1469 cells. miR-152 levels were decreased to 10\% in the NCTC 1469 cells transfected with the miR-152 inhibitor as compared with those transfected with the scramble miRNA. (B) Downregulation of miR-152 inhibited phosphorylation of AKT and GSK in the NCTC 1469 cells treated with or without glucose. (C) The transfection of miR-152 inhibitor reduced the generation of glycogen in the NCTC 1469 cells treated with or without glucose. Data represent the mean \pm standard error of the mean, $\mathrm{n}=3$ independent experiments. ${ }^{*} \mathrm{P}<0.05$ and ${ }^{* * *} \mathrm{P}<0.01 \mathrm{vs}$. control; ${ }^{\#} \mathrm{P}<0.05$ and ${ }^{\# \#} \mathrm{P}<0.01$ vs. glucose. miR-152, microRNA-152; p-, phosphorylated; GSK, glycogen synthase kinase.

miR-152 inhibitor promotes reduction of glycogen synthesis and impairment of AKT and GSK phosphorylation in hepatocytes. In order to further investigate the effects of miR-152 on glucose-induced reduction of glycogen synthesis, miR-152 inhibitor was transfected into the NCTC 1469 cells. As shown in Fig. 3A, miR-152 levels were decreased to $10 \%$ in the NCTC 1469 cells transfected with the miR-152 inhibitor, as compared with those transfected with scrambled miRNA. Furthermore, downregulation of miR-152 inhibited phosphorylation of AKT and GSK in NCTC 1469 cells treated with or without glucose (Fig. 3B). Furthermore, the transfection of miR-152 inhibitor decreased the production of glycogen in NCTC 1469 cells treated with or without glucose (Fig. 3C).

Upregulation of miR-152 reverses the glucose-induced decrease in glycogen synthesis and AKT and GSK phosphorylation in hepatocytes. miR-152 mimic was transfected into the NCTC 1469 cells for $48 \mathrm{~h}$ and then analyzed by qPCR. qPCR indicated that the levels of miR-152 were increased by $\sim 200$ fold in the NCTC 1469 cells transfected with the miR-152 mimic, as compared with those transfected with the scrambled miRNA (Fig. 4A). Furthermore, the transfection of the miR-152 mimic increased the phosphorylation levels of AKT and GSK, and rescued the effects of high glucose on the activation of the AKT/GSK pathway (Fig. 4B). Finally, it was identified that upregulation of miR-152 could reverse the glucose-induced decrease in glycogen synthesis in hepatocytes (Fig. 4C).

\section{Discussion}

Increasing evidence has indicated that miRNA is involved in the pathogenesis of type 2 diabetes and insulin resistance (14). In the present study, it was identified that the activation of AKT and GSK, and the levels of glycogen were inhibited in NCTC 1469 cells and mouse primary hepatocytes, following exposure to $25 \mathrm{mM}$ glucose for $48 \mathrm{~h}$. Furthermore, to the best of our knowledge this study demonstrated for the first time, that high glucose levels suppressed the expression of miR-152 in hepatocytes. In order to further assess the effects of miR-152 on the glucose-induced reduction of glycogen synthesis and activation of AKT and GSK, the miR-152 mimic and inhibitor were transfected into the NCTC 1469 cells, respectively. The results suggested that miR-152 could regulate the activation of $\mathrm{AKT}$ and GSK, and subsequently modulate glycogen synthesis in the NCTC 1469 cells treated with $25 \mathrm{mM}$ glucose for $48 \mathrm{~h}$.

Insulin resistance is a diminished capacity for insulin to stimulate glucose uptake and glycogen synthesis in peripheral tissues, including skeletal muscle, adipose tissue and liver. It is a critical factor in the pathogenesis of type 2 diabetes. The liver has a central role in glucose and lipid metabolism, and hepatic insulin resistance is a hallmark feature of type 2 diabetes. It has been previously reported 
A

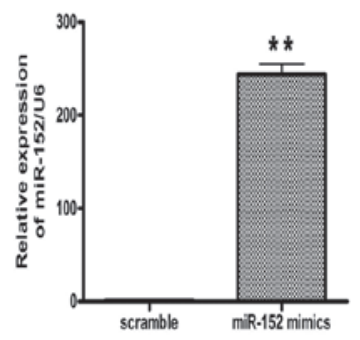

C

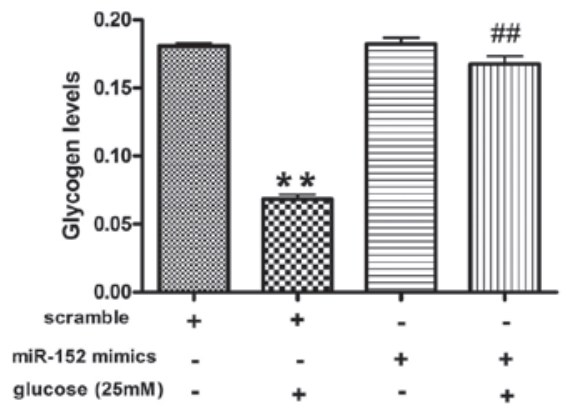

B

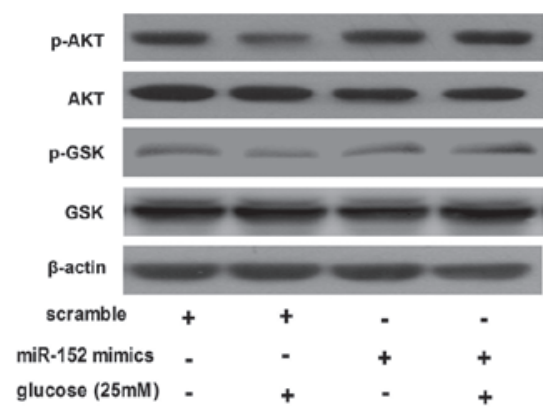

Figure 4. Upregulation of miR-152 reversed the glucose-induced decrease in glycogen synthesis and reduced AKT and GSK phosphorylation in the hepatocytes. miR-152 mimic wastransfectedintotheNCTC1469 cellsfor $48 \mathrm{~h}$.(A)As shownbyquantitative polymerasechainreaction,thelevels ofmiR-152 wereincreased to 200 fold in the NCTC 1469 cells transfected with the miR-152 mimic compared with those transfected with the scramble miRNA.(B) The transfection of miR-152 mimic increasedphosphorylationlevels of AKTandGSK, andrescued theeffectsofhighglucoseontheactivationoftheAKT/GSKpathway.(C)UpregulationofmiR-152could reversethe glucose-induced decrease in glycogen synthesis in the hepatocytes. Data represent the mean \pm standard error of the mean, $n=3$ independent experiments ${ }^{* *} \mathrm{P}<0.01$ versus control; ${ }^{\# \#} \mathrm{P}<0.01$ versus glucose. miR-152, microRNA-152; p-, phosphorylated; GSK, glycogen synthase kinase.

that high glucose-induced oxidative stress is important in the development and progression of hepatic insulin resistance. Furthermore, high glucose has been shown to activate the protein kinase $\mathrm{C}$ and c-Jun $\mathrm{N}$-terminal kinase pathways, which act on the Ser307 phosphorylation of insulin receptor substrate 1 and block the downstream activation of the AKT pathway in the liver $(11,12,15)$. In the liver, the PI3K/AKT pathway functions in the insulin signaling cascade, whereby activated AKT mediates the phosphorylation and inactivation of GSK, which subsequently results in the activation of GS and increased glycogen synthesis. The resulting hyperglycemia is an important factor in the pathogenesis of insulin resistance. Therefore, a high glucose-induced hepatic insulin resistance cell model was used in the present study. Under high glucose conditions, insulin fails to activate its signaling pathway, resulting in an insulin resistant state. Hepatic insulin resistance can be determined by measuring the insulin-mediated phosphorylation of AKT and GSK, and expression levels of glycogen. The present study identified that the levels of phosphorylation of AKT and GSK were reduced, followed by impaired glycogen synthesis, in NCTC 1469 cells and mouse primary hepatocytes, following exposure to $25 \mathrm{mM}$ glucose for $48 \mathrm{~h}$. These results indicate that high glucose levels induced a reduction in glycogen synthesis in hepatocytes through impairing the phosphorylation of AKT and GSK.

To investigate the mechanisms underlying the high glucose-induced reduction of glycogen synthesis and impaired phosphorylation of AKT and GSK in hepatocytes, the effects of high glucose levels on the expression of miR-152 were investigated. The results demonstrated that the expression of miR-152 was downregulated in NCTC 1469 cells treated with $25 \mathrm{mM}$ glucose for $48 \mathrm{~h}$. Similarly, high glucose levels suppressed the expression of miR-152 in mouse primary hepatocytes. These data indicate that miR-152 is involved in glucose-induced insulin resistance.

It has been previously considered that miR-152 is involved in various carcinomas. In human gastric and colorectal cancer, expression levels of miR-152 were significantly lower, as compared with matched non-tumor adjacent tissues (16). In cholangiocarcinoma, interleukin-6 was shown to regulate the expression of miR-152, thus linking inflammation-associated cytokines with oncogenesis in cholangiocarcinoma (17). Tsuruta et al (18) reported that miR-152 expression was decreased in human endometrial cancer, while the restoration of miR-152 expression in endometrial cancer cell lines was sufficient to inhibit tumor cell growth in vitro and in vivo. Furthermore, it was identified that the DNMT-1, E2F3, MET and Rictor genes were candidate targets of miR-152, and the data further suggested that epigenetic silencing could drive endometrial carcinogenesis (18). DNMT-1 is the most abundant methyltransferase in mammalian cells, and functions in the maintenance of DNA methylation. Hypermethylation at promoter $\mathrm{CpG}$ islands and inactivation of multiple tumor suppressor genes are common in carcinomas, and contribute to tumor growth. DNMT-1 has a role in the establishment and regulation of tissue-specific patterns of methylated cyto- 
sine residues $(19,20)$. In cancer cells, reduced expression of miR-152 directly modulated the expression of DNMT-1 (21). Alteration in DNA methylation modulates the expression of specific oncogenes and tumor suppressor genes, leading to carcinoma growth $(22,23)$. However, the involvement of miR-152 in glycogen metabolism remained unclear. To clarify this, miR-152 inhibitor and mimic were transfected into NCTC 1469 cells, respectively. The transfection of miR-152 inhibitor reduced the generation of glycogen, accompanied by impaired phosphorylation of AKT and GSK in NCTC 1469 cells treated with or without glucose. By contrast, upregulation of miR-152 through transfection of miR-152 mimic could reverse the glucose-induced decrease in glycogen synthesis and reduce AKT and GSK phosphorylation in hepatocytes. These data indicate that high glucose levels reduce hepatic glycogenesis by suppressing miRNA-152, which modulates the AKT/GSK pathway, and in turn results in insulin resistance, thereby miR-152 and the AKT-GSK pathway may act as novel therapeutic targets in hepatic glycogenesis.

\section{Acknowledgements}

This study was supported by funding from the National Basic Research Program of China (grant no. 2012CB517502) and the National Natural Science Foundation of China (grant nos. 81270887 and 81070634).

\section{References}

1. Meshkani R and Adeli K: Hepatic insulin resistance, metabolic syndrome and cardiovascular disease. Clin Biochem 42: 1331-1346, 2009.

2. Shearn CT, Fritz KS, Reigan P and Petersen DR: Modification of Akt 2 by 4-hydroxynonenal inhibits insulin-dependent Akt signaling in HepG2 cells. Biochemistry 50: 3984-3996, 2011.

3. Henriksen EJ and Dokken BB: Role of glycogen synthase kinase-3 in insulin resistance and type 2 diabetes. Curr Drug Targets 7: 1435-1441, 2006.

4. Schinner S, Scherbaum WA, Bornstein SR and Barthel A: Molecular mechanisms of insulin resistance. Diabet Med 22: 674-682, 2005.

5. Nawano M, Oku A, Ueta K, et al: Hyperglycemia contributes insulin resistance in hepatic and adipose tissue but not skeletal muscle of ZDF rats. Am J Physiol Endocrinol Metab 278: E535-E543, 2000.

6. Ramachandran D, Roy U,Garg S, et al: Sirtl and mir-9 expression is regulated during glucose-stimulated insulin secretion in pancreatic $\beta$-islets. FEBS J 278: 1167-1174, 2011.

7. Joglekar MV, Parekh VS and Hardikar AA: Islet-specific microRNAs in pancreas development, regeneration and diabetes. Indian J Exp Biol 49: 401-408, 2011.
8. Poy MN, Hausser J, Trajkovski M, et al: miR-375 maintains normal pancreatic alpha- and beta-cell mass. Proc Natl Acad Sci USA 106: 5813-5818, 2009.

9. Kornfeld JW, Baitzel C, Könner AC, et al: Obesity-induced overexpression of miR-802 impairs glucose metabolism through silencing of Hnflb. Nature 494: 111-115, 2013.

10. Chen Y, Song Y, Wang Z, et al: Altered expression of MiR-148a and MiR-152 in gastrointestinal cancers and its clinical significance. J Gastrointest Surg 14: 1170-1179, 2010.

11. Zhang WY, Lee JJ, Kim Y, et al: Amelioration of insulin resistance by scopoletin in high-glucose-induced, insulin-resistant HepG2 cells. Horm Metab Res 42: 930-935, 2010.

12. Nakatani Y, Kaneto H, Kawamori D, et al: Modulation of the JNK pathway in liver affects insulin resistance status. J Biol Chem 279: 45803-45809, 2004.

13. Ramey G, Deschemin JC and Vaulont S: Cross-talk between the mitogen activated protein kinase and bone morphogenetic protein/hemojuvelin pathways is required for the induction of hepcidin by holotransferrin in primary mouse hepatocytes. Haematologica 94: 765-772, 2009.

14. Mao Y, Mohan R, Zhang S and Tang X: MicroRNAs as pharmacological targets in diabetes. Pharmacol Res 75: 37-47, 2013.

15. Nakajima K, Yamauchi K, Shigematsu S, et al: Selective attenuation of metabolic branch of insulin receptor down-signaling by high glucose in a hepatoma cell line, HepG2 cells. J Biol Chem 275: 20880-20886, 2000.

16. Chen Y, Song Y, Wang Z, et al: Altered expression of MiR-148a and MiR-152 in gastrointestinal cancers and its clinical significance. J Gastrointest Surg 14: 1170-1179, 2010.

17. Braconi C, Huang N and Patel T: MicroRNA-dependent regulation of DNA methyltransferase-1 and tumor suppressor gene expression by interleukin-6 in human malignant cholangiocytes . Hepatology 51: 881-890, 2010.

18. Tsuruta T, Kozaki K, Uesugi A, et al: MiR-152 is a tumor suppressor microRNA that is silenced by DNA hypermethylation in endometrial cancer. Cancer Res 71: 6450-6462, 2011.

19. Hino R, Uozaki H, Murakami N, et al: Activation of DNA methyltransferase 1 by EBV latent membrane protein $2 \mathrm{~A}$ leads to promoter hypermethylation of PTEN gene in gastric carcinoma. Cancer Res 69: 2766-2774, 2009.

20. Kang MY, Lee BB, Kim YH, et al: Association of the SUV39H1 histone methyltransferase with the DNA methyltransferase 1 at mRNA expression level in primary colorectal cancer. Int $\mathrm{J}$ Cancer 121: 2192-2197, 2007.

21. Ji W, Yang L, Yuan J, et al: MicroRNA-152 targets DNA methyltransferase 1 in NiS-transformed cells via a feedback mechanism. Carcinogenesis 34: 446-453, 2013.

22. Huang J, Wang Y, Guo Y and Sun S: Down-regulated microRNA-152 induces aberrant DNA methylation in hepatitis B virus-related hepatocellular carcinoma by targeting DNA methyltransferase 1. Hepatology 52: 60-70, 2010.

23. Braconi C, Huang $\mathrm{N}$ and Patel T: MicroRNA-dependent regulation of DNA methyltransferase-1 and tumor suppressor gene expression by interleukin-6 in human malignant cholangiocytes Hepatology 51: 881-890, 2010. 